\title{
Are Podcasts the New Radio? Thresholds in the Macro-Environment
}

\begin{abstract}
This paper considers the podcast and the current status of its disruptive relationship to traditional forms of audio entertainment. The tremendous growth of podcast adoption has yet to develop into substantial growth in revenues as the billion dollar profits of linear radio broadcasters continue to grossly dwarf the monetization structures of even the most frequently downloaded podcasts. While the self-organization and immaterial labour driving the podcast moment could be described as a challenge to the capacities of capital, this paper follows political economist Vincent Mosco's lead of moving beyond discontinuities between old and new communication technologies to evenly attend to their continuities as well. I argue that while the unpaid labour collected into most podcasts seems to locate these cultural products outside of capitalist relations, the uniquely intimate affect podcasts generate represents a continuity between digital and linear audio media as it is captured by capital in the form of both targeted-and influencer-marketing from merchants and brand building social strategies of established broadcasters. Tracing continuities and discontinuities between podcasts and radio, this paper concludes that podcasts should not be considered an over-the-top disrupter to terrestrial radio. It is too soon to predict the degree to which they will enter this role in the future.
\end{abstract}

KEYWORDS: podcast; communication technology; radio; disruption; immaterial labour

a

October 2014 saw the release of Serial, a program from the producers of WBEZ Chicago's This American Life that became a certified hit in the digital realm. Strikingly, Serial is not a video series, but a podcast, a digital audio product that with an average of 1.26 million downloads per episode (Gamerman, Wall Street Journal, 13 November 2014) is quickly approaching the level of audience reach usually reserved for digital video. In terms of the podcasting world in general, Serial has generated an unprecedented level of response, becoming the fastest podcast to reach five million downloads in history (Gamerman 2014). 
Serials whirlwind success has caused managers in digital and traditional media to reappraise the podcast's potential as a major contender for coveted advertising dollars in the world of traditional radio broadcasting, an industry whose national networks continue to draw $\$ 1.15$ billion in ad revenue annually according to Andy Fixmer and Rodney Yap (2013: 28). The main question this paper will address is this: do podcasts constitute a kind of over-the-top in the business of audio-only products, a threat to linear, terrestrial radio analogous with the threat that services like Netflix and Shomi are posing to the billion dollar industry of traditional linear television? My approach is guided by political economist Vincent Mosco's claim that studying the implications of transitions from old to new media requires a skeptical optic that accounts for both the "continuities" between mediums, such as the ways in which new communications technologies replicate and expand preexisting commodity-structures, and the "discontinuities" between them, such as the ways in which new communications technologies pose challenges to the capacities and legal regimes of capitalism (2009: 120-21). Through situating the thresholds between podcasts and linear radio, this paper will address the ways in which managers and producers in podcasting are uniquely advantaged and disadvantaged in our contemporary media environment while providing a foundation for critically attending to these questions of continuity and disjunction between these two communication technologies.

\section{Traditional Dollars, Digital Cents}

When it comes to the question of whether or not podcasting constitutes a formidable threat to traditional radio broadcasting, there are a steady supply of considerations available to dampen the most stalwart confidence. Even with the low cost of production and distribution, the current position of CPMs (cost per thousand viewers) in the ad market for podcasting suggest that a podcast producer who manages to scale to 400,000 net subscribers (translating roughly to three million monthly downloads) can expect to generate enough revenue to break even (Chicago Public Media 2014). This leaves the producer with the essentially freemium approaches of merchandizing, subscription fees and appeals to donors for creating profit and equity in a podcast network business.

While the considerable stir around Serial is stoking optimism about the commercial potential of the overall podcast ecosystem, confidence in the medium's ability to displace traditional radio must be sobered by its dismal track record thus far. eMarketer editorial director Mike Chapman prematurely predicted that advertising revenue for podcasting would hit $\$ 80$ million in 2006 and reach $\$ 300$ million by 2010 (Anderson 2006: 16). This optimistic forecast proved to be a gross overestimation as podcast advertising sales have stagnated at $\$ 34$ million since 2010 (Fixmer and Yap 2013: 28). To put this in perspective, Internet radio outlets like Pandora secured $\$ 331$ million in 2014, and even that number looks negligible when it is placed next to the $\$ 1.15$ billion in ad sales of traditional over-the-air radio networks. This 
disparity can be at least partially accredited to the self-activity and self-organization associated with podcasts. The degree of choice and diversity podcasts deliver is driven by a flurry of volunteer, creative labour chiefly organized outside of capital, and the gap in revenues between podcasting and other audio businesses suggest that the immaterial nature of this activity, like other forms of immaterial labour identified by scholars in the autonomist school (Mosco 2009: 121), prevents the value it generates from being straightforwardly captured by capital. But while the economics of immaterial labour may be coming to bear, the slight revenues of podcasting can also be explained with the mere circumstance that podcasting, unlike linear radio, is not a mature business. The disruptive possibilities of podcasting rest on its capacity to seize market shares from traditional radio.

Eroding the gigantic revenues that traditional radio broadcasting has enjoyed uninterrupted for decades will be no small feat, and based on recent numbers by audience measurement and information company Nielsen, this project has not been going well. Nielsen's second quarter 2014 report entitled Shift in Viewing suggests that consumption of terrestrial radio is still going strong. According to the report, adults aged eighteen and over spend an average of two hours and forty-five minutes per day listening to AM/FM radio, making radio the second most widely consumed medium next to linear television (Nielsen 2014: 8). The report includes measurements for time spent using the internet on a computer, using a smartphone and even using a video game console, but, perhaps tellingly, it does not contain a category for time spent listening to podcasts.

Despite this, recent technological developments suggest that the audience reach of podcasts is poised for growth. A major leap for podcasting is thought to have come from a recent update to the iPhone's iOS operating system, which included a podcast app. In the past, podcast listeners had to download podcasts using their computers and then sync their devices to those computers before they could enjoy these products. Now, iPhone users need only open an app to access endless amounts of free, audio content available to be downloaded directly to their device. This has dramatically changed the consumption of podcasting, as 50 per cent of the total podcasts being downloaded today are on a smartphone device (Wolf 2013). Apple's CarPlay — an iPhone-like touchscreen interface released last year that can be installed directly into the built-in displays in a lineup of luxury and economy vehicles - promises to stimulate more growth as it eliminates another step for the millions of commuters who listen to podcasts while they travel. As the experience of listening to podcasts becomes more seamless, the car-being one of the last places in which people listen to the radio-will become a crucial beachhead for managers of digital audio content as they continue to compete with traditional forms of radio broadcasting.

These trends are apparently in agreement with Nielsen's Senior Vice President of Insights, Dounia Turrill, who forecasts significant changes to the current media 
landscape driven by the growth of digital audiences (Nielsen 2014: 1). But the extraordinary degree to which media managers in podcasting have been forced to discount their ad revenues and mortgage their creative labour suggests that even as podcasts continue to gain more dissemination with popular audiences, they may, along with digital video, find themselves caught up in a trend of trading analogue dollars for digital cents, to offer a variation on Jeff Zucker's extensively quoted phrase (Albrecht 2009).

\section{The Intimacy Advantage}

While monetization opportunities for podcasting remain low, an advantage that could convert into higher revenues in the future is thought to come from the intensely affective nature of the podcast medium. Podcasts constitute a resource in the digital world that, along with mobile devices, e-mail, blogs, digital video and social networking sites, have altered totally the nature of direct selling relationships between the manufacturer, the distributor and the consumer (Ferrell et al. 2010: 159). Embedded in this process, advertising in podcasts reads less like ads and more like sponsorships. In comedy podcasts, the ads are humorous and are often seamlessly integrated into the content of the show. In general, podcast ads are more effective than online keyword and display ads because they capitalize on a listener's natural ability to comprehend oral communication, making the transmission instinctual in a way that reading and writing do not (Tulley 2011: 258). The medium is also more intimate than other forms of digital media, with listeners building strong relationships with podcast hosts as they literally allow those people into their ears on a weekly basis. Podcast ads appear less crassly commercial than radio ads in that they are often read by the podcast host in a conversational manner, presenting the listener with an opportunity to indirectly support an entrepreneurial individual who is offering content for free. In most cases, podcast hosts will not align themselves with a sponsor unless they've tried out the product or service and can vouch for its quality and value, giving a brand a stamp of approval to a small but intensely loyal audience who trusts that person (Kristin 2014: 33). Igloo Software's Creative Director Marko Savic, a routine advertiser on a selection of podcasts, discusses this element of intimacy when he cites the podcast's "captive audience," which he believes drove 7 per cent of his company's opportunities while influencing the quality and value of its total opportunities by 21 per cent in 2013 (Lex 2014).

The intimate nature of podcasts is at the core of why director-digital of Palisades MediaGroup Mike Laughlin has recently argued that buying podcast ads is "five times as effective as buying one website" (Riismandel 2014). The affective sociality created by podcasts make them attractive modes of advertising for brands coveting these high quality, engaged, dedicated audiences. While at present total annual ad revenues in podcasting make just under 3 per cent of the total annual advertising revenues of terrestrial radio, it is a consolation that powerful brands are evidently very hungry for the captive audiences that podcasts consistently deliver, with blue 
chip accounts like Ford Motors, 21st Century Fox, and Walt Disney's ABC beginning to invest in the medium alongside smaller merchants like Igloo and Frank and Oak (Fixmer and Yap 2013: 28).

\section{Benefits for Broadcasters}

Since podcasts are trickier to monetize, the benefits of investing in podcasts for established radio broadcasters both public and private lie entirely in the ability of a podcast to strengthen brand power. Podcasting has recently moved into the arena of social media, making it an indispensable part of a multi-platform digital buy (Obar et al. 2013: 43). Next to internet video, which is currently experiencing a growth of 50.2 per cent, podcasts represent the second most valuable form of new media in terms of "sharing product and brand experiences" (Ferrell et al. 2010: 159) with a growth rate of 29.6 per cent. Serial is a spinoff from another podcast perpetually dominating the iTunes charts, called This American Life, which is essentially a re-broadcast of a show produced by WBEZ Chicago and distributed through the Public Radio Exchange (PRX) that, in addition to its 800,000 subscribers on iTunes, continues to be delivered to between 1.7-1.8 million listeners through traditional linear radio (Wolf 2013). Host Ira Glass remarks that the podcast, not the radio, has been the main way in which the audience of This American Life has grown over the past decade (Wolf 2013).

In terms of the Canadian context, the Canadian Broadcasting Corporation (CBC) has especially benefited from this ability to re-broadcast content through the web, with its podcasts routinely ranking in the iTunes top twenty and reaching one million monthly downloads in 2013 (Obar et al. 2013: 35-6). When considering president Hubert Lacroix's plan to de-emphasize the CBC's linear radio and television offerings while prioritizing its mobile/digital services, there is an additional strategic value of continuing to develop high-quality audio content that lies in that content's capacity to hit two birds with one stone: On the one hand, podcasts are versatile, digital products consumed by a steadily growing international listenership. On the other, such an investment could fulfill the CBC's mandate to make its services accessible to Canadians who do not have access to tablets or smartphones, since the same content can be delivered over its radio infrastructure.

As the podcast industry develops, the brands of $\mathrm{CBC}$ and other public broadcasters may also benefit from the nature of podcast advertising, which can be framed as a corporate sponsorship or alignment rather than the more devalued category of the radio ad. Public broadcasters that claim to be ad free, such as TVOntario, will still make allowances for corporate sponsorships as a revenue stream (TVO 2014: 25). Likewise, Serial is produced by a noncommercial organization and distributed through PRX, but it still draws a large degree of financial support from the sponsorship of an email-marketing service called MailChimp (Gamerman 2014). 


\section{Conclusion}

In sum, this paper has surveyed a number of continuities and discontinuities between terrestrial radio and podcasts in order to evaluate the disruptive potential of the podcast medium. Despite the extent to which the podcast industry has grown, the epidemically insubstantial advertising revenues still require even the most popular podcast personalities to turn to Kickstarter and listener donations to make ends meet. But while the commercial feasibility of the podcast for managers in digital media warrants skepticism, the benefits for advertisers are not in question as the intimate nature of the podcast and the tailored demographic of its audiences create excellent returns for merchants. For traditional public and private broadcasters, podcasts present an opportunity to remain relevant and bolster brand presence, but it comes at the price of potentially cannibalizing the exceedingly more lucrative ad revenues of terrestrial radio. Examining the liminality between radio and podcasts in these various areas brings to attention a challenge to traditional modes of capitalist extraction in the communications and entertainment sectors, one presented by the largely self-organized labour that has both spread the podcast into virtually every segment of the population and made it decidedly difficult to commercialize. However, the affective sociality podcasts foster also creates opportunities to replicate and deepen capitalist relations, evinced in the ways in which advertisers and broadcasters differently draw on and exploit the intimate networks of podcast listenerships. Taking all of these arguments together, the extent to which the podcast will disrupt the industry is uncertain. Perhaps the true mark of the podcast's shift into the position of a formidable competitor to AM/FM radio will be a mention in Nielsen's next quarterly report.

\section{References}

Albrecht, Chris. 2009. Zucker: We're at Digital Dimes Now. Gigaom, 18 March. https:// gigaom.com/ 2009/03/18/zucker-were-at-digital-dimes-now/.

Anderson, Diane. 6 March 2006. Podcasting Ads Reel in $\$ 80$ Million. Brandweek 47(10): 16.

Chicago Public Media. 2014. It's Not the Product, It's the Person. This American Life, August 5. http://www.thisamericanlife.org/radio-archives/episode/533/transcript.

Ferrell, Linda, Tracy L. Gonzalez-Padron and O.C. Ferrell. 2010. An Assessment of the Use of Technology in the Direct Selling Industry. Journal of Personal Selling \& Sales Management 30(2): 157-65.

Fixmer, Andy and Rodney Yap. 9 December 2013. Celebrity Podcasts Are Fueling a Second Act. Bloomberg Businessweek (4358): 28-28.

Friedman, Lex. 2014. Case Study: Igloo Software and The Mid Roll. Midroll. http:// www.midroll.com/advertisers/.

Gamerman, Ellen. 2014. “Serial” Podcast Catches Fire. Wall Street Journal, 13 November. http:// www.wsj.com/articles/serial-podcast-catches-fire-1415921853. 
Laird, Kristin. 2014. What Men Want. Marketing 119(3): 28-33.

Mosco, Vincent. 2009. The Political Economy of Communications. 2nd Edition. London: Sage.

Obar, Jonathan A., Gregory Taylor, Derek Antoine, Rena Bivens, Nadia Caidi, Arndis Johnson, Catherine A. Middleton and David Skinner. 2013. Mapping Digital Media: Canada. SSRN. http://papers.ssrn.com/abstract $=2455895$

Riismandel, Paul. 2014. Recent Press for Midroll and the Business of Podcasting. Midroll. http://www.midroll.com/great-press-midroll-business-podcasting/.

Nielsen. 2014. Shift in Viewing: The Cross-Platform Report Q2 2014. The Cross-Platform Series, 8 September.

Tulley, Christine. 2011. IText Reconfigured: The Rise of the Podcast. Journal of Business and Technical Communication 25(3): 256-75.

TVO. 2014. TVO Annual Report 2013-2014. TVO. http://about.tvo.org/sites/default/ files/TVO_AnnualReport_2013-14_English_AODA.pdf.

Wolf, Michael. 2013. Funnymen and iPhones: Why the Podcast Is Finally Coming Into Its Own. Forbes, 4 April. http://www.forbes.com/sites/michaelwolf/2013/04/04/ funnymen-and-iphones-why-the-podcast-is-finally-coming-into-its-own/. 